\title{
INHALT DES ZWEITEN HEFTES
}

\section{Aufsätze (Articles)}

\section{Hans Hermann Spoo}

Die Finanzen des Rasselstein in den Jahiren 1823-24. Ein Beitrag zur Frühgeschichte

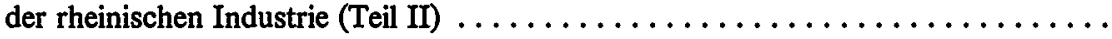
The Finance of Rasselstein in 1823-24. A Contribution to the History of Early Industrialization in the Rhineland (Part II)

\section{Hubert Kiesewetter}

„Mein Vater ist mit seinen McCormick Ma(s)chinen sehr zufrieden.“ Verkaufsstrategien eines internationalen Unternehmens in Deutschland . . . . . . . . . . . . „My father is very satisfied with his McCormick machines.“ Selling strategies of an international U.S. enterprise in Germany

\section{Bibliographie (Bibliography)}

Martina Mundorf unter Mitarbeit von Petra Kirste Bibliographie zur Unternehmensgeschichte und Unternehmerbiographie (Berichtsjahr

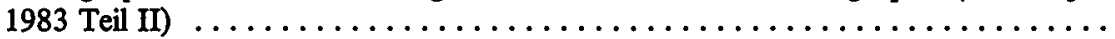
Short Bibliography on Business History and Entrepreneurial Biography (1983 Year Report Part II)

\section{Buchbesprechungen (Reviews)}

Jürgen Mura (Bearb.), Der öffentliche Auftrag der Sparkassen in der historischen Entwick-

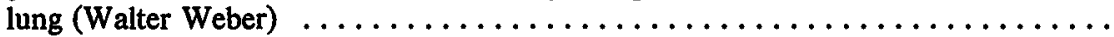

Wilhelm Treue, Geschichte einer Hamburgischen Anwaltssozietät (Maria Möring) . . . Harald Pohlmann, Richard C. Krogmann 1859-1933 (Lars U. Scholl) . . . . . . . . . Gunter Gach, In Schacht und Strecke (Carl-Friedrich Baumann) . . . . . . . . . . . Hans Pohl, Hegenscheidt: Von Ratibor nach Erkelenz (Konrad Fuchs) . . . . . . . . . Hans Konradin Herdt, Bosch 1886-1986

Robert Bosch 1861-1942, Bosch 1886-1986 (Toni Pierenkemper) . . . . . . . . . 138

Stefan Karner, Die Steiermark im Dritten Reich 1938-1945 (Lothar Höbelt) . . . . . 139

Alois Brusatti/Verena Hofstätter, Produktinnovationen (Franz Mathis) . . . . . . . 140

Alois Brusatti/Hans Pohl (Hrsg.), Unternehmen und Staat nach dem Zweiten Weltkrieg

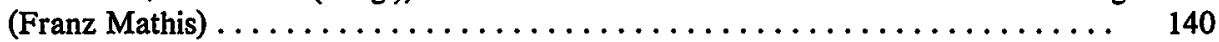

Volker Hentschel, Wirtschaftsgeschichte des modernen Japans, 2 Bde (Franz Mathis) . 141 Hans-Peter Merz/Sung-Jo Park, Japanisches Management in der Bundesrepublik

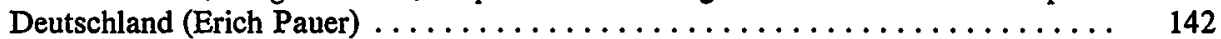

Mitteilungen (Informations) $\ldots \ldots \ldots \ldots \ldots \ldots \ldots \ldots \ldots \ldots \ldots \ldots \ldots \ldots \ldots \ldots$ 